\title{
Cyp26b1 Within the Growth Plate Regulates Bone Growth in Juvenile
}

\section{Mice}

Yoshiki Minegishi ${ }^{\mathrm{a}, \mathrm{b}, \mathrm{c}}$, Yasuo Sakai ${ }^{\mathrm{c}, \mathrm{d}}$, Yasuhito Yahara ${ }^{\mathrm{a}}$, Haruhiko Akiyama ${ }^{\mathrm{e}}$, Hideki

Yoshikawa $^{\mathrm{f}}$, Ko Hosokawa $^{\mathrm{c}}$, and Noriyuki Tsumakia, ${ }^{\mathrm{a}, \mathrm{g}}$

${ }^{a}$ From the Department of Cell Growth and Differentiation, Center for iPS Cell Research and Application, Kyoto University, 53 Kawahara-cho, Shogoin, Sakyo-ku, Kyoto 606-8507, Japan

${ }^{b}$ Department of Plastic and Reconstructive Surgery, University of Fukui Hospital, 23-3

Matsuokashimoaizuki, Eiheiji-cho, Yoshida-gun, Fukui 910-1193, Japan

${ }^{c}$ Department of Plastic Surgery, Osaka University Graduate School of Medicine, 2-2 Yamadaoka, Suita, Osaka 565-0871, Japan

${ }^{\mathrm{d} D e p a r t m e n t ~ o f ~ P l a s t i c ~ S u r g e r y, ~ B e l l l a n d ~ G e n e r a l ~ H o s p i t a l, ~ 500-3 ~ H i g a s h i y a m a ~ N a k a-k u, ~}$ Sakai, Osaka 599-8247, Japan

${ }^{\text {e}}$ Department of Orthopaedic Surgery, Gifu University Graduate School of Medicine, 1-1 Yanagito, Gifu 501-1194, Japan

fDepartment of Orthopaedic Surgery, Osaka University Graduate School of Medicine, 2-2 Yamadaoka, Suita, Osaka 565-0871, Japan

gJapan Science and Technology Agency, CREST, Tokyo, 102-0075, Japan

To whom correspondence should be addressed:

Noriyuki Tsumaki, Department of Cell Growth and Differentiation, Center for iPS Cell Research and Application, Kyoto University, 53 Kawahara-cho, Shogoin, Sakyo-ku, Kyoto 606-8507, Japan, Tel: +81-75-366-7045; Fax: +81-75-366-7047; E-mail: ntsumaki@cira.kyoto-u.ac.jp 


\section{Abstract}

Retinoic acid (RA) is an active metabolite of vitamin A and plays important roles in embryonic development. CYP26 enzymes degrade RA and have specific expression patterns that produce a RA gradient, which regulates the patterning of various structures in the embryo. However, it has not been addressed whether a RA gradient also exists and functions in organs after birth. We found localized RA activities in the diaphyseal portion of the growth plate cartilage were associated with the specific expression of Cyp26b1 in the epiphyseal portion in juvenile mice. To disturb the distribution of RA, we generated mice lacking Cyp26b1 specifically in chondrocytes $\left(C y p 26 b 1^{\text {Achon }} \mathrm{cKO}\right)$. These mice showed reduced skeletal growth in the juvenile stage. Additionally, their growth plate cartilage showed decreased proliferation rates of proliferative chondrocytes, which was associated with a reduced height in the zone of proliferative chondrocytes, and closed focally by four weeks of age, while wild-type mouse growth plates never closed. Feeding the Cyp26b1 cKO mice a vitamin A-deficient diet partially reversed these abnormalities of the growth plate cartilage. These results collectively suggest that Cyp26b1 in the growth plate 
regulates the proliferation rates of chondrocytes and is responsible for the normal function of the growth plate and growing bones in juvenile mice, probably by limiting the RA distribution in the growth plate proliferating zone.

Keywords: retinoic acid; growth plate; Cyp26b1; chondrocytes

Abbreviations: RA, retinoic acid; RARE, RA-responsive elements; Cyp26b1 ${ }^{4 c h o n} \mathrm{cKO}$, 11Enh-Cre; Cyp26b floxflox conditional knockout 


\section{Introduction}

Retinoic acid (RA) is an active metabolite of vitamin A and plays important roles in embryonic development. The concentration of RA is controlled by the balance between its synthesis by retinaldehyde dehydrogenase (RALDH) and its degradation by CYP26 enzymes. CYP26s are a group of P450 enzymes that metabolize RA to inactive forms [1,2,3]. Both RALDH and Cyp26 have specific expression patterns and produce a RA gradient [4]. This RA gradient regulates the patterning of the anterior-posterior axis of various structures, including the hindbrain and paraxial mesoderm [5]. The RA gradient also regulates the proximodistal patterning and outgrowth of the developing limbs. Cyp26b1 is expressed in the distal region of developing limb buds, and mice that lack Cyp26b1 show severe limb malformation due to the spreading of the RA signal toward the distal end of the developing limb, causing abnormal patterning of limb skeletal elements [6].

However, whether this RA gradient also regulates the growth and maintenance of organs after birth has not been addressed.

The growth plate cartilage is where bone growth occurs in juveniles. Growth plate cartilage is located in the metaphysis at each end of long bones. Chondrocytes residing at the epiphyseal side in the growth plate cartilage proliferate and subsequently undergo 
hypertrophy just after stopping proliferation. As a result, chondrocytes change from proliferating chondrocytes on the epiphyseal side to hypertrophic chondrocytes on the diaphyseal side of growth plate cartilage. The hypertrophic chondrocytes residing at the diaphyseal end of the growth plate cartilage subsequently die, and the hypertrophic cartilage is degraded and gradually replaced by bone. Through this mechanism, the bone becomes elongated. Thus, strict regulation of chondrocyte proliferation and hypertrophic differentiation is necessary for the normal growth of bones.

In juveniles, focal closures of the growth plate in the distal tibia [7], the proximal tibia [8] , the distal tibia, elbow, proximal femur and distal femur [9] are caused by the treatment of acne with retinoids [7] or the treatment of hyperkeratinosis with cis-retinoic acid $[8,9]$. In guinea pigs, the application of RA caused closure of the growth plates in the proximal tibia [10]. These clinical and preclinical manifestations indicate that vitamin A and its metabolites play important roles in the bone growth of juveniles. The RA signal has been shown to regulate bone growth after birth. The deletion of RA receptors (RAR) in chrondocytes disturbs skeletal growth due to abnormal chondrocyte differentiation and disturbed matrix synthesis within the growth plates in mice [11]. Additionally, a localized distribution of RA was detected in the growth plate cartilage of the ribs of three-week-old 
rabbits [12]. However, it remains to be determined how RA regulates the bone growth of juveniles. In this study, we found that the expression of Cyp26b1 specifically in the proliferative chondrocyte zone was associated with localized RA activities in the zone of hypertrophic chondrocytes in the growth plate cartilage of juvenile mice. To disturb the distribution of RA, we inactivated Cyp26b1 in the growth plate using Cyp26b1 conditional knock-out mice. The resulting mouse phenotype suggests that Cyp26b1 within the growth plate regulates the proliferation rates of chondrocytes and bone growth in juveniles. 


\section{Materials and Methods}

\subsection{Animals and PCR genotyping procedures}

RARE-LacZ mice were a gift from Dr. Janet Rossant [13]. To generate Cyp26b1 conditional knockout mice, 11Enh-Cre transgenic mice [14,15] and Cyp26blflox/flox mice [16] were prepared and mated to generate 11Enh-Cre; Cyp26b1 flox/+ mice. Then, the 11Enh-Cre; Cyp26b1 $1^{\text {flox/+ }}$ and Cyp26b1 flox/flox mice were intercrossed, and the 11Enh-Cre; Cyp26b1 flox/flox mice were considered conditional knockout $\left(C y p 26 b 1^{\text {Achon }} \mathrm{cKO}\right)$ mice. The 11Enh-Cre; Cyp26b1 flox/+ mice were used as controls.

For genotyping, genomic DNA was isolated from the tail tips or embryonic skin and subjected to PCR analysis, according to a previously described method for the Cre transgene [14] and Cyp26b1 allele [16].

\subsection{Frozen sectioning and laser capture microdissection (LMD)}

Mouse hindlimbs were harvested without fixation and were immediately embedded in SCEM compound (SECTION-LAB, Hiroshima, Japan). Frozen sections were prepared at 6- $\mu \mathrm{m}$ thickness with a Cryofilm type 2c(9) (SECTION-LAB) using a CM3050S cryomicrotome (Leica), according to the method described by Kawamoto [17]. The 
sections were briefly fixed with $100 \%$ ethanol. Semiserial sections were then stained with hematoxylin and eosin.

For LMD, frozen sections were prepared with LMD film (SECTION-LAB) using a cryomicrotome. The sections were freeze-dried in the cryostat chamber at $-25{ }^{\circ} \mathrm{C}$ for one hour and briefly fixed with $100 \%$ ethanol. The proliferative zone and hypertrophic zone in the growth plate were individually captured and microdissected from cryosections using a Leica LMD7000 device (Leica) and put onto the dip of the lid of $0.5 \mathrm{ml}$ tubes with cold TRIzol (Life Technologies, Tokyo, Japan).

\subsection{Real-time RT-PCR}

RNA was extracted from the collected samples using RNeasy Mini Kits (Qiagen, Tokyo, Japan). The total RNA was digested with DNase to eliminate any contaminating genomic DNA. For real-time quantitative RT-PCR analyses, $1 \mu \mathrm{g}$ of total RNA was reverse-transcribed into first-strand cDNA using ReverTra Ace (Toyobo, Osaka, Japan) and random primers. The PCR amplification was performed in a reaction volume of $20 \mu \mathrm{l}$ containing $2 \mu \mathrm{l}$ of cDNA, $10 \mu \mathrm{l}$ of SYBR FAST qPCR Master Mix (Kapa Biosystems, Tokyo, Japan) and 7900HT (Applied Biosystems). The RNA expression levels were 
normalized to the level of Gapdh expression. The primers used are listed in Table 1.

\subsection{Staining of the skeleton}

Mouse limbs were dissected, fixed in $100 \%$ ethanol overnight and then stained with Alcian blue, followed by Alizarin red S solution, according to standard protocols [18].

\subsection{Histological analysis}

Mouse limbs were dissected, fixed in 4\% paraformaldehyde, processed and embedded in paraffin. For the immunohistochemical analysis, sections were incubated with an anti-CYP26B1 antibody (Scrum Inc., Tokyo, Japan) and an anti-type X collagen antibody (COSMO BIO CO., LTD.,Tokyo, Japan). Immune complexes were detected using secondary antibodies conjugated to Alexa Fluor 555 and Alexa Fluor 546, respectively.

\subsection{BrdU staining}

Mice were intraperitoneally injected with $\mathrm{BrdU}$ labeling reagent (10 $\mu \mathrm{l} / \mathrm{g}$ body weight)

(Zymed Laboratories Inc., South San Francisco, CA) two hours before being sacrificed. The mice were then dissected and sectioned. The incorporated BrdU was detected using a 
BrdU staining kit (Zymed Laboratories, Inc., South San Francisco, CA) to distinguish actively proliferating cells. The mean number of BrdU-positive cells/total cells \pm standard deviation (S.D.) was calculated.

\subsection{Microscope}

Images were acquired on an inverted microscope (Eclipse Ti; Nikon) equipped with cameras (DS-Fi1; Nikon and C4742-80-12AG; Hamamatsu photonics) and the NIS Elements software program (Nikon).

\subsection{Statistical analyses}

Data are shown as the means and S.D. Student's $t$-test was used to compare data. P values $<0.05$ were considered statistically significant. 


\section{Results}

3.1 Localized expression of Cyp26b1 and regulation of RA activities along the direction of bone elongation within the growth plate cartilage.

To test our hypothesis that a RA gradient exists within the growth plate cartilage, we employed RARE-lacZ transgenic mice, in which lacZ is expressed under the control of RA-responsive elements (RARE) [13]. Because trabecular bone shows high background galactosidase activities, it is difficult to detect transgene-specific lacZ activities in bone, especially after birth. Therefore, we microdissected the growth plate cartilage, obtained cells from the proliferative chondrocyte and hypertrophic zones (Figure 1A), and subjected them to an analysis of the expression of the lacZ transgene mRNA by real-time RT-PCR. The expression analysis of proliferative and hypertrophic chondrocyte markers confirmed that cells were isolated appropriately by microdissection (Figure 1B). LacZ was much more highly expressed in the hypertrophic chondrocytes than in the proliferative chondrocytes (Figure 1B). Interestingly, we detected a much higher level of Cyp26b1 mRNA expression in the proliferative chondrocytes than in the hypertrophic chondrocytes (Figure 1B). Accordingly, immunohistochemical analysis showed that CYP26B1 was expressed more abundantly in proliferative chondrocytes than in hypertrophic chondrocytes (Figure 1C). 
These results suggest that regulated RA distribution along the direction of the bone elongation exists within the growth plate cartilage and is generated by the exclusive localization of CYP26B1 in the proliferative chondrocyte zone (Figure 1D).

\subsection{Cyp26b1 deletion in chondrocytes disturbs the bone growth in juveniles}

To disturb the distribution of RA, we deleted Cyp26b1 in the proliferative chondrocytes of the growth plate cartilage by generating 11Enh-Cre; Cyp26b floxflox conditional knockout (Cyp26b1 $\left.1^{\text {Achon }} \mathrm{cKO}\right)$ mice. The 11Enh-Cre transgene directed Cre expression in the proliferative chondrocytes after the completion of skeletal patterning under the control of the promoter/enhancer sequence of the type XI collagen $\alpha 2$ chain gene (Coll1a2) [14,15]. Cyp26b1 $1^{4 c h o n} \mathrm{cKO}$ mice showed normal skeletal patterning, skeletal development and bone length until one week after birth. However, at three weeks after birth, they started to show a chondrodysplasia phenotype that included dwarfism and a short snout (Figures 2A and 2B). A precise examination of the lengths of skeletal elements revealed a gradual decrease in the lengths of bones compared to control mice beginning from two weeks after birth (Figures 2C-E), and all long bones in the Cyp26b1 $1^{\Delta c h o n} \mathrm{cKO}$ mice were shorter than those in the controls at three weeks after birth. 
To analyze the mechanism responsible for the shorter bones in the Cyp26b1 ${ }^{4 c h o n} \mathrm{cKO}$ mice, we performed a histological analysis of their growth plates. We noticed a slight decrease in the height of the proliferative chondrocyte zone at two weeks after birth (Figure $3 \mathrm{~A}$, top row). The height of the proliferative chondrocyte zone was significantly decreased at three weeks after birth, especially at the central portion (Figure 3A, middle row, and Figure 3B). Immunohistochemistry using an anti-type $\mathrm{X}$ collagen antibody revealed that the location of the hypertrophic cartilage zone that produced type $\mathrm{X}$ collagen was shifted toward the epiphysis in Cyp26b1 cKO mice, clarifying the decreased height of the zone of proliferative chondrocytes (Figure $3 \mathrm{C}$ ) to the central portion.

The growth plate was closed at the central portion at four weeks after birth (Figure $3 \mathrm{~A}$, bottom row), and growth plate closure was recognized in all bones examined, including the tibia, humerus and femur (Figures 3A, D). BrdU labeling analysis revealed significantly decreased proliferation rates of the proliferative chondrocytes when the mice were one and three weeks old (Figures 4A-C). These results suggest that the decreased proliferation rates of the proliferative chondrocytes can explain the decreased heights of the proliferative chondrocyte zone, the subsequent closure of the growth plates and the suppression of bone growth. 
3.3. Reversion of the growth plate abnormalities by feeding Cyp26b1 ${ }^{4 c h o n} c K O$ mice a vitamin A-deficient diet

The immunohistochemical analysis confirmed that CYP26B1 was absent in the proliferative chondrocytes of Cyp26b1 $1^{4 c h o n} \mathrm{cKO}$ mice (Figure 4D), and that this absence could cause a disturbance in the RA distribution within the growth plate.

To confirm that the elevated concentration of RA in the proliferative chondrocytes of Cyp26b1 $1^{4 c h o n} \mathrm{cKO}$ mice was responsible for the decreased proliferation rates, we fed the mice a vitamin A-deficient diet which systemically decreased RA concentrations [19]. Following this diet, Cyp26b1 $1^{4 c h o n} \mathrm{cKO}$ mice showed a partial reversion in the decreased proliferation rates of the proliferative chondrocytes (Figures 4E) and corresponding partial reversion in the decreased height of the proliferative chondrocyte zone (Figure 4F). These results collectively suggest that the distribution of RA, which is generated by the proliferative chondrocyte-specific expression of Cyp26b1, regulates the proliferation rates of chondrocytes and subsequently controls the growth rates and closure of growth plates to determine the length of bones in juveniles. 


\section{Discussion}

It is well known that the RA gradient regulates the patterning of spatial structures in mammals during development. Cyp26b1 is a critical regulator of the distribution of RA, and Cyp26b1 KO and cKO studies have demonstrated that Cyp26b1 deletion alters the RA distribution in various tissues $[5,16,20,21]$. The present study provides evidence that Cyp26b1 within the growth plate cartilage is important for normal chondrocyte proliferation/differentiation and bone growth in juvenile mice, probably through its regulation of the RA distribution in the growth plate.

We detected localized RA activities within the growth plate cartilage in juvenile mice, as indicated by the increased $R A R E-l a c Z$ expression toward hypertrophic chondrocytes

(Figure 1B). The existence of a possible RA gradient within the growth plate is supported by a previous report demonstrating that the RA content measured by MS quantification was lower in the resting and proliferative chondrocyte zone than that in the hypertrophic chondrocyte zone in the growth plate cartilage of the ribs of three-week old rabbits [12]. It was reported that $R A R E-l a c Z$ expression is not detectable or is limited in the primordial cartilage during the embryonic stage [22], which is consistent with the normal embryonic development of Cyp26b1 ${ }^{4 c h o n} \mathrm{cKO}$ mice in this study. 
The partial recovery of growth plate abnormalities in Cyp26b1 $1^{\Delta c h o n} \mathrm{cKO}$ mice by a vitamin A-deficient diet supports the notion that Cyp26b1 deletion caused abnormalities through elevated RA activities in the growth plate. Accordingly, the decreased proliferation rates of proliferative chondrocytes in Cyp26b1 $1^{4 c h o n} \mathrm{cKO}$ mice suggest that excess RA inhibits the proliferation of proliferative chondrocytes. Previous studies on the effects of RA have yielded conflicting data regarding its regulation of the differentiation of chondrocytes in the growth plate [23]. Ballock et al. reported that RA blocks the stimulatory effects of thyroid hormone on cultured rat chondrocyte hypertrophy [23], whereas others showed RA induces the hypertrophy of cultured chick chondrocytes $[24,25]$. Such in vitro culture experiments are affected by the pharmacological dosage of RA, variations in the species, culture conditions, composition of serum and the anatomical source of the growth plate cells used [23]. Our in vivo results are less susceptible to the above problems to better clarify the function of RA in chondrocyte proliferation/differentiation in the growth plate and in the bone growth.

Excess intake of vitamin A causes growth impairment and skeletal pain in juveniles $[7,8,9]$. Our study provides evidence that regulation of the RA distribution occurs within the growth plate cartilage and that this regulation plays critical roles in controlling the 
growth of long bones in juvenile mice. Our results also show that exogenous vitamin A can affect this regulation and distribution, since a vitamin A-deficient diet partially rescued the growth plate structure of Cyp26b1 $1^{4 c h o n} \mathrm{cKO}$ mice. The characteristics of the closure of the growth plate seen in a child receiving excess RA [8] and guinea pigs receiving a RAR agonist [10] were similar to those seen in $C y p 26 b 1^{4 c h o n} \mathrm{cKO}$ mice in that the closure occurred in the central part of the growth plate, suggesting that a similar form of regulation occurs in humans and mice.

\section{Acknowledgements}

We thank Hiroshi Hamada and Satoru Mamiya for the Cyp26b1 flox mice, Aki Takimoto,

Chisa Shukunami and Yuji Hiraki for instructions about the cryostat sectioning, Janet

Rossant for the RARE-LacZ mice, Peter Karagiannis for reading the manuscript, and Hiromi Kishi, Minoru Okada, Akihiro Yamashita and Miho Morioka for assistance and helpful discussions. This study was supported in part by the Japan Science Technology Agency (JST), CREST (to N.T.), Scientific Research Grant No. 23792042 (to Y. M.) and 
No. 24390354 (to N.T.) from MEXT. 


\section{References}

[1] H. Fujii, T. Sato, S. Kaneko, O. Gotoh, Y. Fujii-Kuriyama, K. Osawa, S. Kato, H. Hamada, Metabolic inactivation of retinoic acid by a novel P450 differentially expressed in developing mouse embryos, Embo j 16 (1997) 4163-4173.

[2] W.J. Ray, G. Bain, M. Yao, D.I. Gottlieb, CYP26, a novel mammalian cytochrome P450, is induced by retinoic acid and defines a new family, J Biol Chem 272 (1997) 18702-18708.

[3] J.A. White, Y.D. Guo, K. Baetz, B. Beckett-Jones, J. Bonasoro, K.E. Hsu, F.J. Dilworth, G. Jones, M. Petkovich, Identification of the retinoic acid-inducible all-trans-retinoic acid 4-hydroxylase, J Biol Chem 271 (1996) 29922-29927.

[4] S. Shimozono, T. Iimura, T. Kitaguchi, S. Higashijima, A. Miyawaki, Visualization of an endogenous retinoic acid gradient across embryonic development, Nature 496 (2013) 363-366.

[5] Y. Sakai, C. Meno, H. Fujii, J. Nishino, H. Shiratori, Y. Saijoh, J. Rossant, H. Hamada, The retinoic acid-inactivating enzyme CYP26 is essential for establishing an uneven distribution of retinoic acid along the anterio-posterior axis within the mouse embryo, Genes Dev 15 (2001) 213-225. 
[6] K. Yashiro, X. Zhao, M. Uehara, K. Yamashita, M. Nishijima, J. Nishino, Y. Saijoh, Y. Sakai, H. Hamada, Regulation of retinoic acid distribution is required for proximodistal patterning and outgrowth of the developing mouse limb, Dev Cell 6 (2004) 411-422.

[7] F. Luthi, Y. Eggel, N. Theumann, Premature epiphyseal closure in an adolescent treated by retinoids for acne: an unusual cause of anterior knee pain, Joint Bone Spine 79 (2012) 314-316.

[8] L.M. Milstone, J. McGuire, R.C. Ablow, Premature epiphyseal closure in a child receiving oral 13-cis-retinoic acid, J Am Acad Dermatol 7 (1982) 663-666.

[9] J. Prendiville, E.A. Bingham, D. Burrows, Premature epiphyseal closure--a complication of etretinate therapy in children, J Am Acad Dermatol 15 (1986) $1259-1262$.

[10] A.M. Standeven, P.J. Davies, R.A. Chandraratna, D.R. Mader, A.T. Johnson, V.A. Thomazy, Retinoid-induced epiphyseal plate closure in guinea pigs, Fundam Appl Toxicol 34 (1996) 91-98.

[11] J.A. Williams, N. Kondo, T. Okabe, N. Takeshita, D.M. Pilchak, E. Koyama, T. Ochiai, D. Jensen, M.-L. Chu, M.A. Kane, J.L. Napoli, M. Enomoto-Iwamoto, N. 
Ghyselinck, P. Chambon, M. Pacifici, M. Iwamoto, Retinoic acid receptors are required for skeletal growth, matrix homeostasis and growth plate function in postnatal mouse., Developmental biology 328 (2009) 315-327.

[12] J.A. Williams, M. Kane, T. Okabe, M. Enomoto-Iwamoto, J.L. Napoli, M. Pacifici, M. Iwamoto, Endogenous retinoids in mammalian growth plate cartilage: analysis and roles in matrix homeostasis and turnover, J Biol Chem 285 (2010) 36674-36681.

[13] J. Rossant, R. Zirngibl, D. Cado, M. Shago, V. Giguere, Expression of a retinoic acid response element-hsplacZ transgene defines specific domains of transcriptional activity during mouse embryogenesis, Genes Dev 5 (1991) 1333-1344.

[14] T. Iwai, J. Murai, H. Yoshikawa, N. Tsumaki, Smad7 Inhibits chondrocyte differentiation at multiple steps during endochondral bone formation and down-regulates p38 MAPK pathways, J Biol Chem 283 (2008) 27154-27164.

[15] D. Ikegami, H. Akiyama, A. Suzuki, T. Nakamura, T. Nakano, H. Yoshikawa, N. Tsumaki, Sox9 sustains chondrocyte survival and hypertrophy in part through Pik3ca-Akt pathways, Development 138 (2011) 1507-1519.

[16] J. Okano, U. Lichti, S. Mamiya, M. Aronova, G. Zhang, S.H. Yuspa, H. Hamada, Y. Sakai, M.I. Morasso, Increased retinoic acid levels through ablation of Cyp26b1 
determine the processes of embryonic skin barrier formation and peridermal development, J Cell Sci 125 (2012) 1827-1836.

[17] T. Kawamoto, Use of a new adhesive film for the preparation of multi-purpose fresh-frozen sections from hard tissues, whole-animals, insects and plants, Arch Histol Cytol 66 (2003) 123-143.

[18] P.W.J. Peters, Double staining of fetal skeletons for cartilage and bone., in: H.J.M.D. Neuberg, T.E. Kwasigroch (Eds.), Methods in Prenatal Toxicology, Georg Thieme Verlag, Stuttgart, Germany, 1977, pp. 153-154.

[19] A.K. Verma, A. Shoemaker, R. Simsiman, M. Denning, R.D. Zachman, Expression of retinoic acid nuclear receptors and tissue transglutaminase is altered in various tissues of rats fed a vitamin A-deficient diet, J Nutr 122 (1992) 2144-2152.

[20] H.J. Dranse, A.V. Sampaio, M. Petkovich, T.M. Underhill, Genetic deletion of Cyp26b1 negatively impacts limb skeletogenesis by inhibiting chondrogenesis, J Cell Sci 124 (2011) 2723-2734.

[21] G. MacLean, H. Li, D. Metzger, P. Chambon, M. Petkovich, Apoptotic extinction of germ cells in testes of Cyp26b1 knockout mice, Endocrinology 148 (2007) $4560-4567$. 
[22] H.P. von Schroeder, J.N. Heersche, Retinoic acid responsiveness of cells and tissues in developing fetal limbs evaluated in a RAREhsplacZ transgenic mouse model, J Orthop Res 16 (1998) 355-364.

[23] R.T. Ballock, X. Zhou, L.M. Mink, D.H. Chen, B.C. Mita, Both retinoic acid and 1,25(OH)2 vitamin D3 inhibit thyroid hormone-induced terminal differentiaton of growth plate chondrocytes, J Orthop Res 19 (2001) 43-49.

[24] M. Iwamoto, I.M. Shapiro, K. Yagami, A.L. Boskey, P.S. Leboy, S.L. Adams, M. Pacifici, Retinoic acid induces rapid mineralization and expression of mineralization-related genes in chondrocytes, Exp Cell Res 207 (1993) 413-420.

[25] M. Pacifici, E.B. Golden, M. Iwamoto, S.L. Adams, Retinoic acid treatment induces type X collagen gene expression in cultured chick chondrocytes, Exp Cell Res 195 (1991) 38-46. 


\section{Figure legends}

Figure 1. Regulated RA distribution along the long axis of long bones within the growth plate cartilage.

(A) We microdissected the growth plate cartilage of the proximal tibia to obtain chondrocytes from the proliferative zone (PCZ) and hypertrophic zone (HCZ) from RARE-LacZ mice at 2.5 weeks after birth. Left, a semiserial section was stained with hematoxylin and eosin. Right, a residual section after microdissection.

(B) RNAs were extracted from cells in the proliferative chondrocyte zone (PCZ) and cells in the hypertrophic chondrocyte zone (HCZ), which were respectively obtained by microdissection, and were subjected to a real-time RT-PCR expression analysis for the genes indicated at the top of each graph. ${ }^{*} \mathrm{P}<0.01(\mathrm{n}=5)$.

(C) Histological sections of growth plate cartilage from the proximal tibias from three-week-old mice were immunostained with an anti-CYP26B1 antibody (Red). Bar: $100 \mu \mathrm{m}$. 
(D)A schematic representation of the RA concentration and Cyp26b1 expression within the growth plate cartilage. Regulated RA activities were found along the long axis of the long bone within the growth plate cartilage and attributed to the localized expression of CYP26B1 in the proliferative chondrocyte zone.

Figure 2. The skeletal phenotype of 11Enh-Cre; Cyp26b1 floxflox $\left(C y p 26 b 1^{\text {Achon }}\right) \mathrm{cKO}$ mice and 11Enh-Cre; Cyp26b1 $1^{\mathrm{flox} /+}$ control mice.

(A) The gross appearances of mice three weeks after birth. Cyp26b1 $1^{4 c h o n} \mathrm{cKO}$ mice exhibited dwarfism with a short snout. Bar: $1 \mathrm{~cm}$.

(B) X-ray images of mice three weeks after birth.

(C) The skeletons of the forelimbs of mice three weeks after birth. Alcian blue/Alizarin red staining. Bar: $5 \mathrm{~mm}$.

(D) The lengths of the tibiae at one, two, three and four weeks after birth. *P $<0.05$; **P $<0.01(\mathrm{n}=5)$.

(E) Lengths of the humeri, femurs and tibiae three weeks after birth. ${ }^{*} \mathrm{P}<0.01(\mathrm{n}=5)$.

Figure 3. Histological analysis of the growth plate cartilage of Cyp $26 b 1^{4 c h o n} \mathrm{cKO}$ mice. 
(A) Sagittal sections of the proximal tibia at two, three and four weeks after birth. Safranin O-fast green-iron hematoxylin staining. Bar: $500 \mu \mathrm{m}$.

(B) Top, Magnified image of the central part of the growth plate cartilage in the proximal tibia three weeks after birth. Safranin O-fast green-iron hematoxylin staining. Yellow bars indicate the height of the proliferative chondrocyte zone. Blue bars denote the height of the hypertrophic chondrocyte zone. Bars: $100 \mu \mathrm{m}$. Bottom, the mean \pm S.D. of the height of the proliferative chondrocyte zone (left) and the hypertrophic chondrocyte zone (right). $* \mathrm{P}<0.01(\mathrm{n}=5)$.

(C) Sagittal sections of the proximal tibia at three weeks after birth were immunostained with an anti-type X collagen antibody. Bars: $100 \mu \mathrm{m}$.

(D)Histology of the growth plate cartilage in the proximal humerus and distal femur in Cyp26b1 $1^{4 c h o n}$ cKO mice six weeks after birth. Safranin O-fast green-iron hematoxylin. Bars: $500 \mu \mathrm{m}$.

Figure 4. Proliferation rates and Cyp26b1 expression of chondrocytes in the growth plate cartilage, and the effects of a vitamin A-deficient diet on the growth plate cartilage in the proximal tibias of Cyp26b1 $1^{\text {Achon }} \mathrm{cKO}$ mice. 
(A, B, C) BrdU was injected intraperitoneally into the mice two hours before sacrifice. One-week old mice (A) and three-week old mice (B). Top, hematoxylin-eosin staining. Bottom, semiserial sections were immunostained with an anti-BrdU antibody. Regions corresponding to the boxed regions in the top are magnified and shown. Bars: $100 \mu \mathrm{m}$. (C) Ratios (\%) of the number of BrdU-positive cells to the numbers of total cells in the proliferative chondrocyte zone. ${ }^{*} \mathrm{P}<0.01(\mathrm{n}=5)$.

(D) Histological sections of the growth plate cartilage in the proximal tibias of three-week-old mice were immunostained with an anti-Cyp26b1 antibody (Red). The blue color is Hoechst stain, which indicates nuclei. Bars: $100 \mu \mathrm{m}$.

(E, F) Effects of a vitamin A-deficient diet on the growth plate cartilage of Cyp26b1 $1^{\text {4chon }}$ cKO mice. The mothers of Cyp26b1 $1^{\Delta c h o n}$ cKO pups were fed with a vitamin A-deficient diet or a control diet from eight weeks before gestation until the sacrifice of the Cyp26b1 $1^{\Delta c h o n}$ cKO pups three weeks after birth. The pups consumed either their mother's milk or shared an identical diet with their mother.

(E) Left, BrdU was injected intraperitoneally into the mice two hours before sacrifice. Top, hematoxylin-eosin staining. Bottom, semiserial sections were immunostained with an anti-BrdU antibody. Regions corresponding to the boxed regions in the top are 
magnified and shown. Bars: $100 \mu \mathrm{m}$. Right, ratios (\%) of the number of BrdU-positive cells to the number of total cells in the proliferative chondrocyte zone. ${ }^{*} \mathrm{P}<0.01$ ( $\mathrm{n}=$ $5)$.

(F) Mean \pm S.D. of the height of the proliferative chondrocyte zone (left) and the hypertrophic chondrocyte zone (right). ${ }^{* *} \mathrm{P}<0.05(\mathrm{n}=5)$. 
Table 1. Primer sequences used in this study.

\begin{tabular}{|c|c|}
\hline Primer & Sequence (5' to 3') \\
\hline Col2a1 S & TTGAGACAGCACGACGTGGAG \\
\hline Col2a1 AS & AGCCAGGTTGCCATCGCCATA \\
\hline Col10a1 S & GGTGTGAATGGGCGGAAAG \\
\hline Col10a1 AS & GCTTCCCAATACCTTCTCGTC \\
\hline MMP13 S & TGTTTGCAGAGCACTACTTGAA \\
\hline MMP13 AS & CAGTCACCTCTAAGCCAAAGAAA \\
\hline Runx2 S & CCGCACGACAACCGCACCAT \\
\hline Runx2 AS & CGCTCCGGCCCACAAATCTC \\
\hline Beta Galactosidase S & CTCAAACTGGCAGATGCACGGT \\
\hline Beta Galactosidase AS & CGTTGCACCACAGATGAAACGC \\
\hline Cyp26b1 S & GCAAGATCCTACTGGGCGAAC \\
\hline Cp26b1 AS & TTGGGCAGGTAGCTCTCAAGT \\
\hline Gapdh $S$ & AAGCCCATCACCATCTTCCAGGAG \\
\hline Gapdh AS & ATGAGCCСТTCCACAATGCCAAAG \\
\hline
\end{tabular}


Fig.1

A

HE

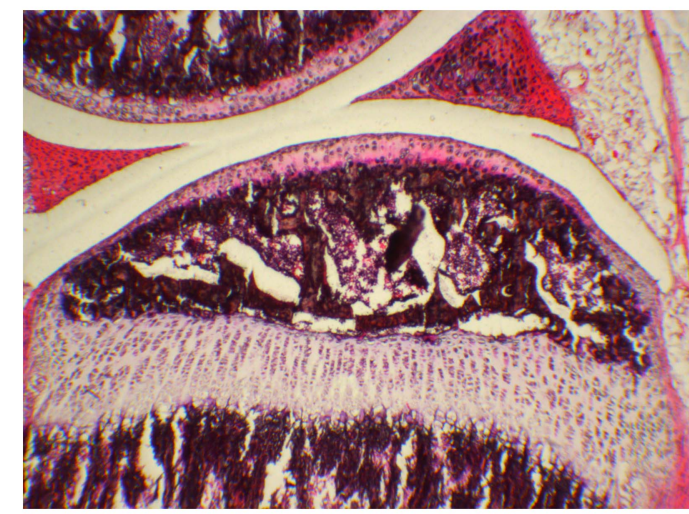

Unstained

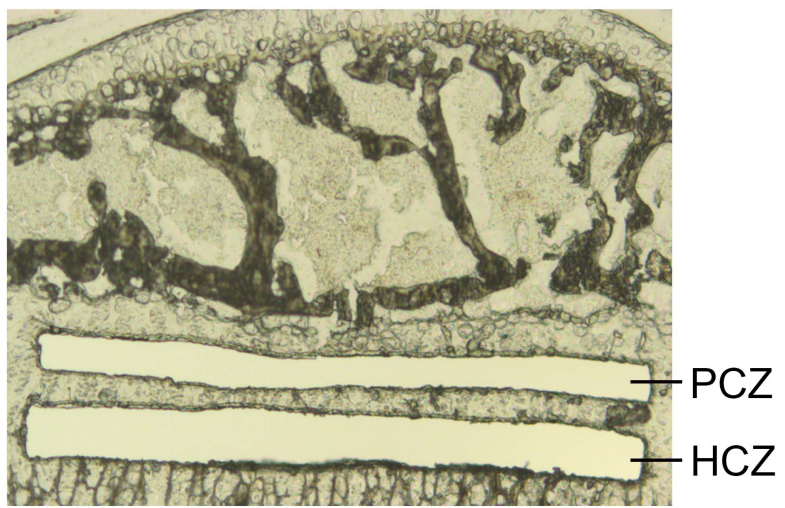

B
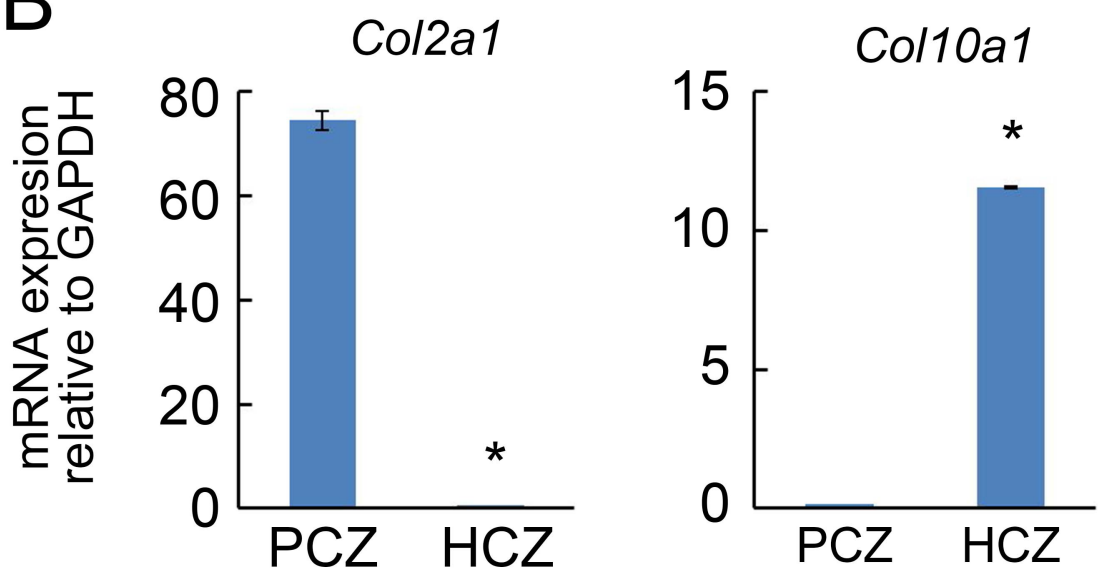

MMP13
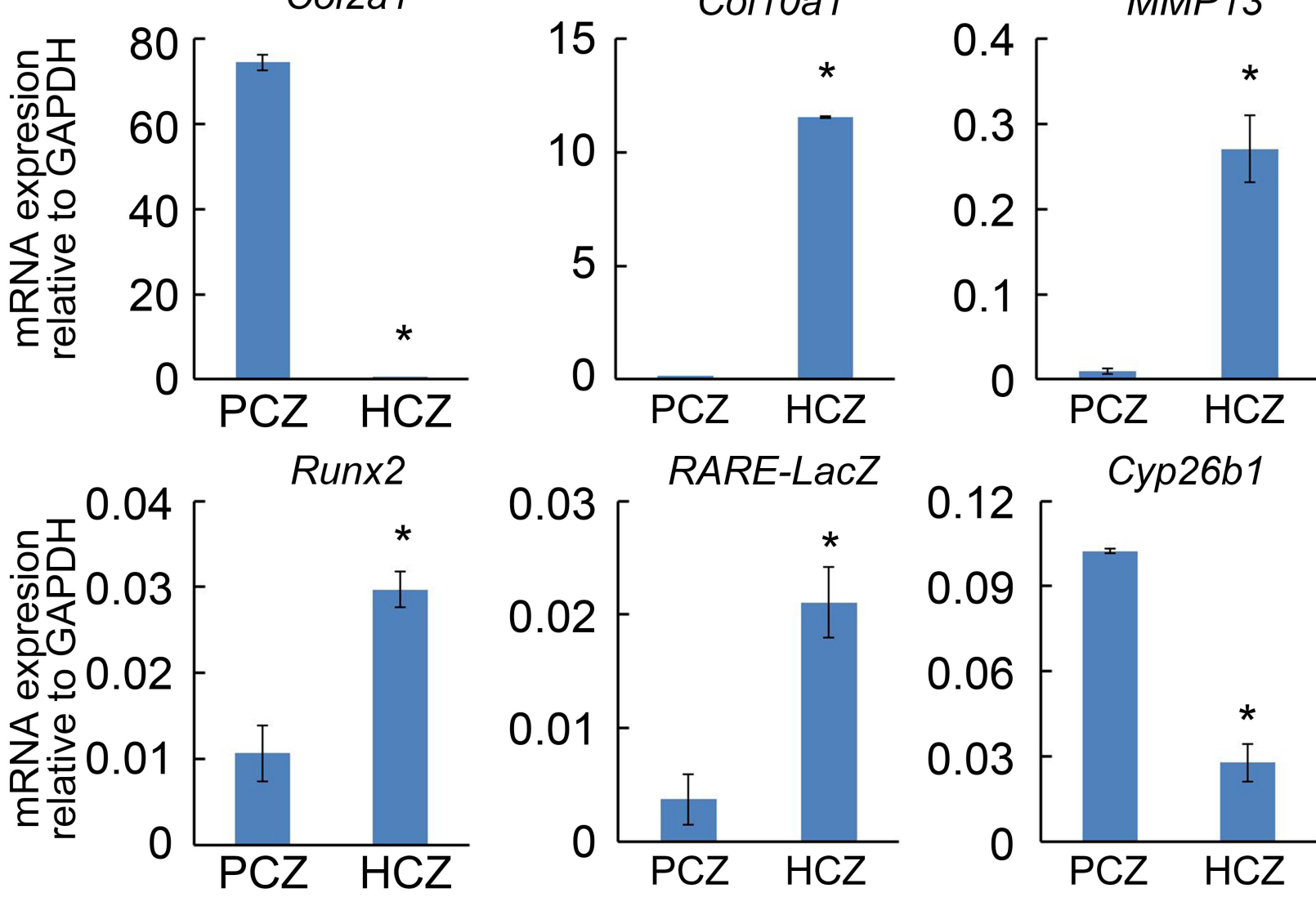

C

D

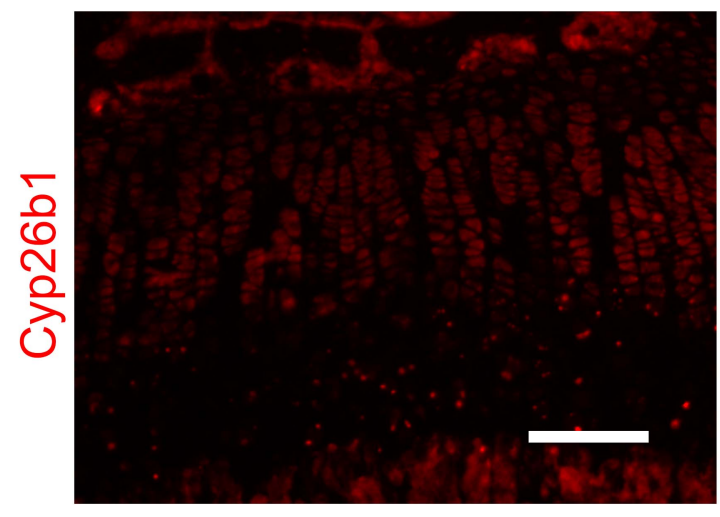

(ㄷ) (2) (2) (2) (2) (2) (2) (ㅇ) (2) Proliferative (0) Proliferative o) (ㅇ () () Chondrocytes 5 (?) (2)

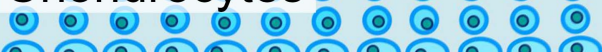
๑ 0.0อง

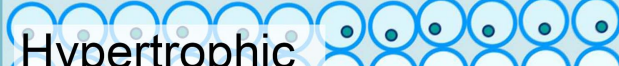
Hypertrophic 060 Chondrocytes 000 Growth Plate 
Fig.2
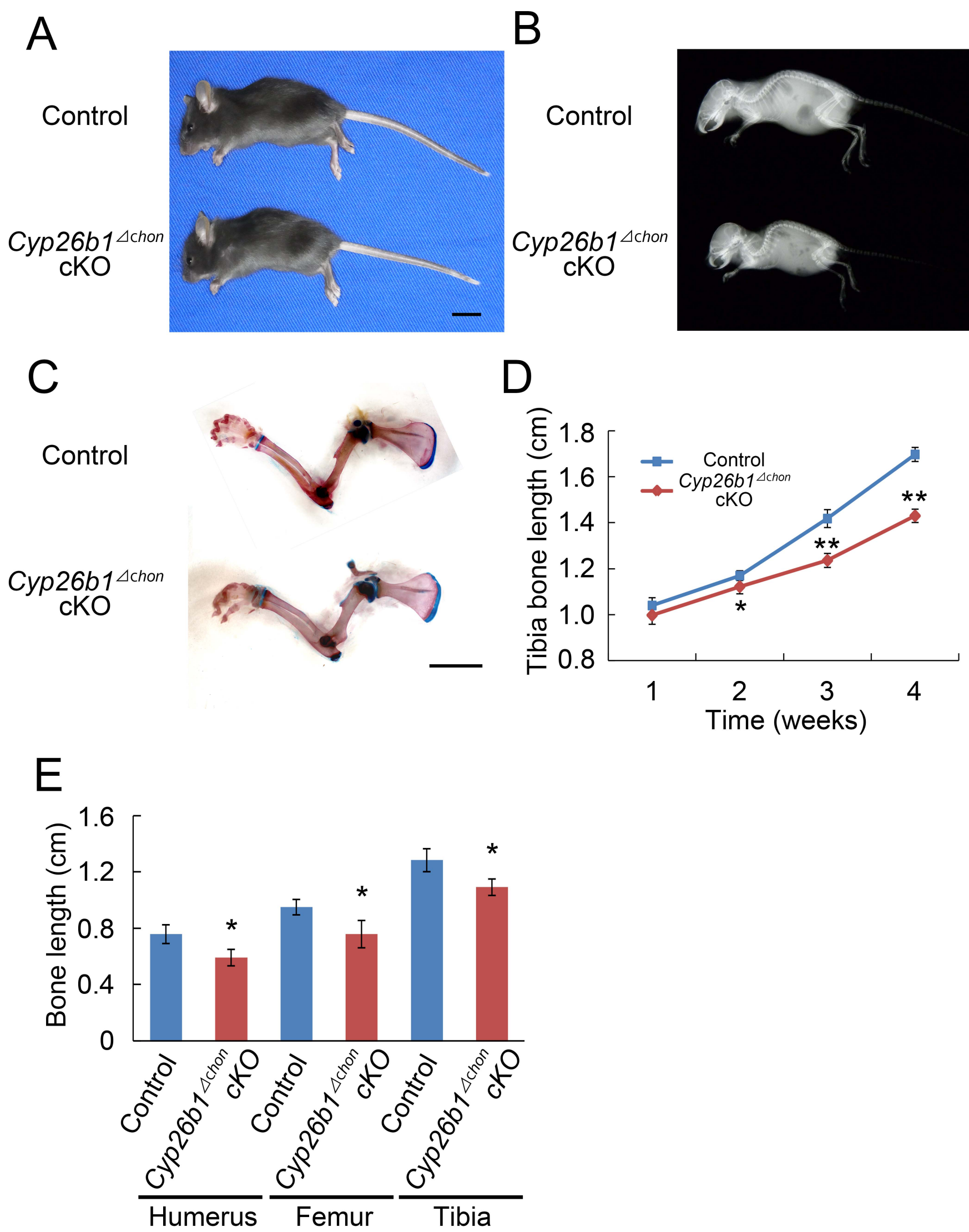
Fig. 3
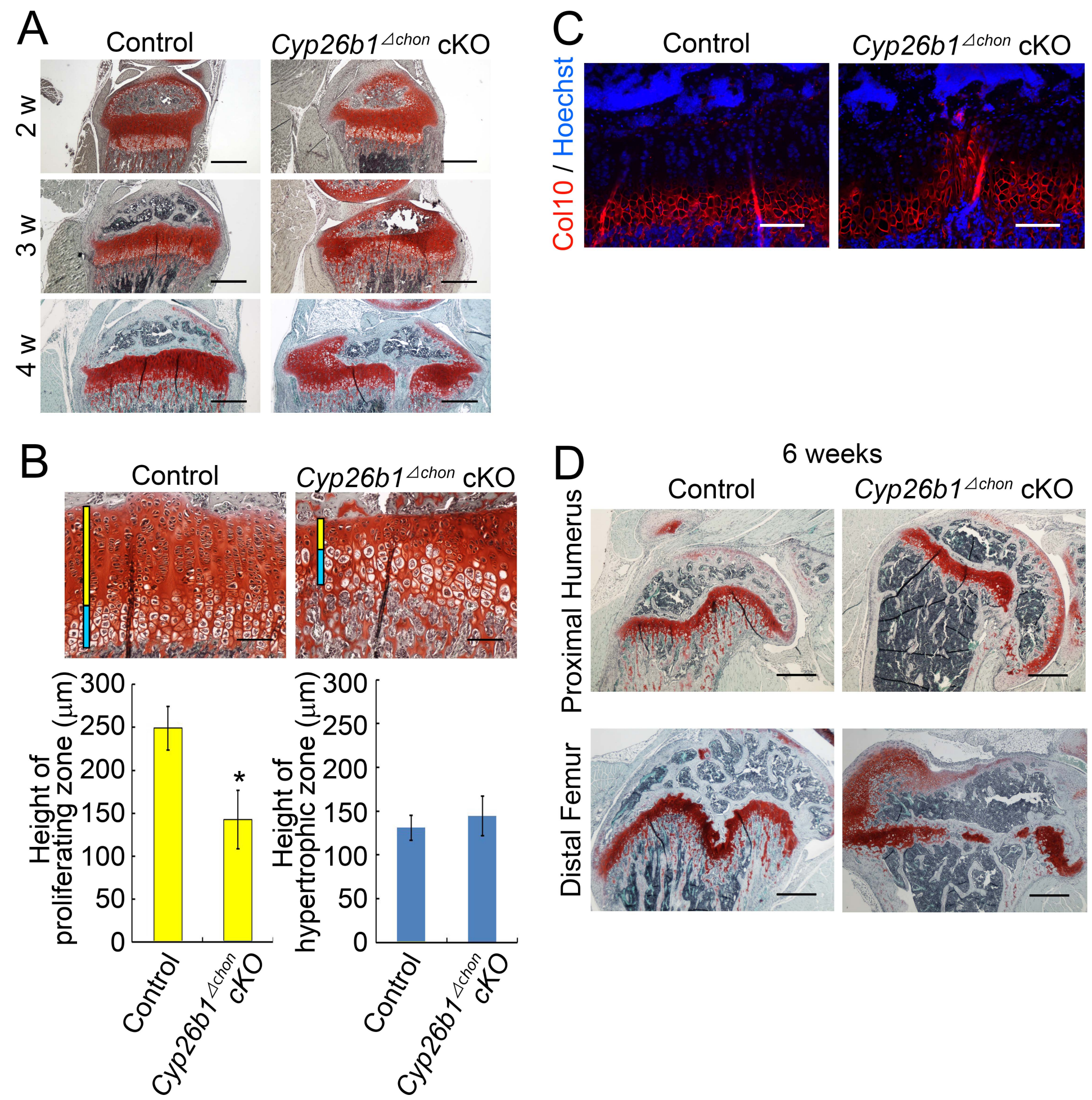
Fig.4
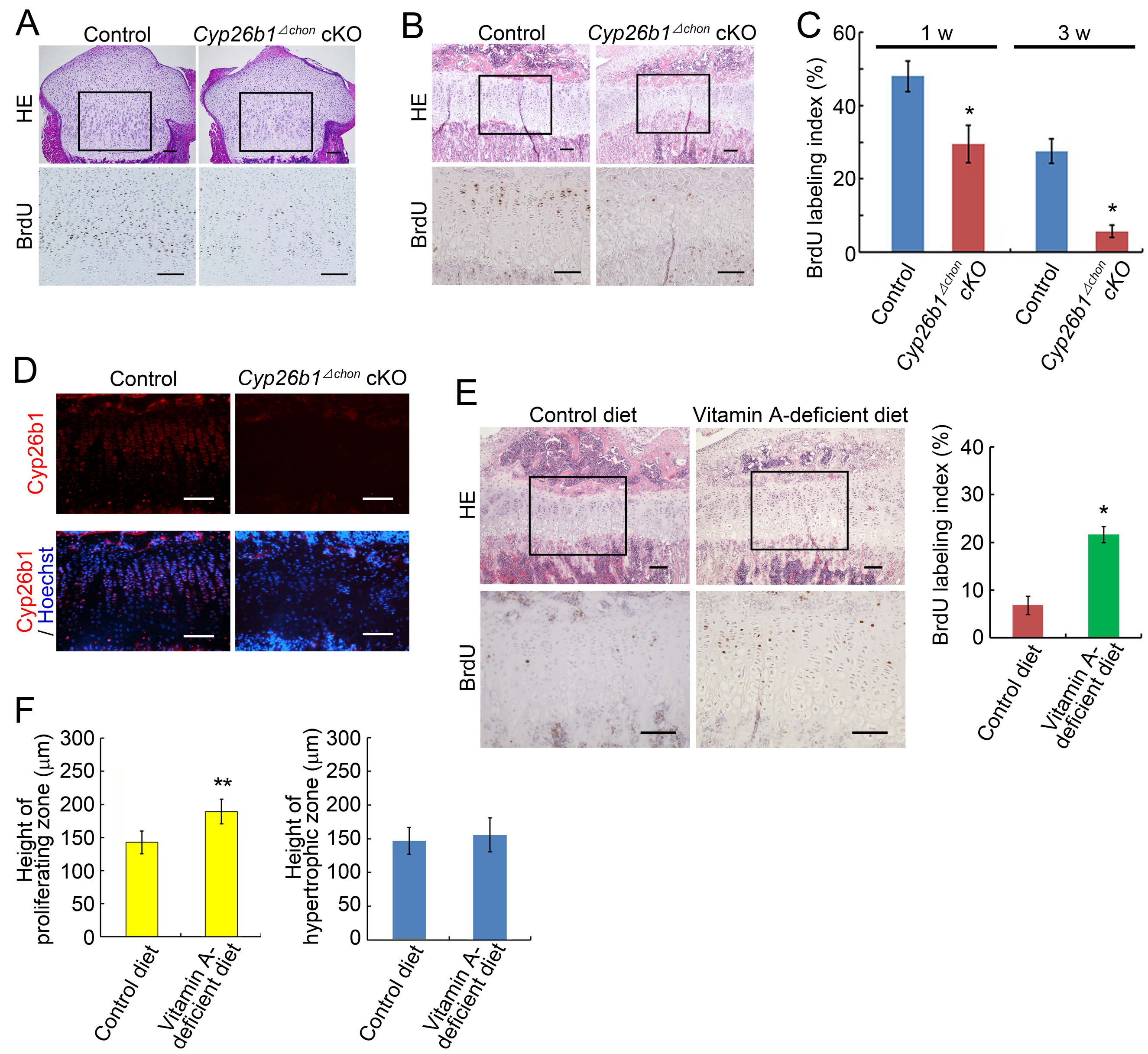